Methods 11 focus groups with the public $(n=36)$, and those recently diagnosed with an STI $(n=20)$, explored perceived barriers and facilitators to using prototype self-sampling packs and returning samples. Using the behaviour change wheel approach to direct intervention development, we engineered an optimised self-sampling and treatment pack and instructions, supported by audio-visual online materials. In this way we translated lay perspectives into evidence-based and theoretically informed, pragmatic recommendations.

Results Using rich participant extracts we illustrate how our analysis suggests: the design of the package should physically separate and order components to be used at each stage in the self-sampling/treatment process; simple written and online audio-visual instructions, suitable for those with low literacy levels should be provided; the rationale for and health consequences of not testing for STIs, including HIV, should be clearly articulated, enabling users to opt-out of HIV testing without inadvertently opting-out of STI testing; specific information concerning the viability of both self-taken samples and postal delivery to laboratories is needed

Conclusion This study represents the first evidence-based approach to improving the design of self-sampling packs and sample return. Using qualitative approaches and implementation science it is possible to systematically suggest refinements to product design and the need for additional sources of psychological and behavioural support to improve user experience, increase acceptability of self-sampling, broaden uptake and boost sample return

Disclosure No significant relationships.

\section{P049 STI TESTING AND DOCUMENTATION VIA A PHONE APPLICATION (APP): EXPERIENCE WITH THE SAFE APP}

${ }^{1}$ Kenneth Fife*, ${ }^{2}$ Ryan Williams, ${ }^{2}$ Angelica Howard, ${ }^{2}$ Gabriella Palmeri, ${ }^{2}$ Lauren Weiniger, ${ }^{3}$ Ken Mayer. 'SAFE Health, Novato, USA; ${ }^{2}$ SAFE Health, Los Angeles, USA; ${ }^{3}$ SAE Health, Los Angeles, USA

\subsection{6/sextrans-2019-sti.254}

Background The STI epidemic continues to grow among young people. Encouraging screening and careful partner selection are approaches to controlling STI transmission.

Methods The SAFE App is a free, phone-based program that encourages users to undergo regular testing for STIs. The app allows for the real-time collection of data concerning the frequency of testing among various demographic populations. Users may securely upload test results from their regular healthcare provider or arrange to be tested through the app. Users who test through the app can use health insurance or pay a flat fee to test without generating an insurance claim. Test results can be securely displayed on the user's phone so they have the ability to easily share them with potential sex partners. The app is available for both Apple and Android systems and is supported by a network of physicians who communicate results to users and arrange for treatment for any user with a positive result. State reporting requirements are also fulfilled through the app.

Results To date, a limited number of users have registered for the app including about 200 from 38 states who imported results into the app and about 150 from 22 states who obtained testing through the app. Among the latter, testing was completed at a commercial laboratory and the result electronically transferred to the SAFE medical record for rapid review by a physician and release to the user. Some users reported that they had never had an STI screen prior to testing through the app. After completion of scalability testing, the app will be promoted through social media.

Conclusion Widespread use of this app should encourage more testing of at-risk populations, generate demographic data concerning the frequency of STI testing, as well as ultimately reducing the spread of STIs by more judicious partner selection.

Disclosure No significant relationships.

\section{P050 SEXUAL HEALTH LONDON ONLINE TESTING: A REVIEW OF SERVICE USERS AND OUTCOMES}

${ }^{1}$ Victoria Tittle, ${ }^{2}$ Tim Alston, ${ }^{2}$ Ryan Kinsella*, 'Sophie Jones, ${ }^{1}$ Sara Day, ${ }^{1}$ David Asboe. ${ }^{1}$ Chelsea and Westminster Hospital NHS Trust, HIVIGU, NH, UK; ${ }^{2}$ Preventx, NH, UK

\subsection{6/sextrans-2019-sti.255}

Background Sexual Health London (SHL) provides online asymptomatic sexual health screen, channelling patients away from clinic attendances. We present data from commencement on the $8^{\text {th }}$ January to $31^{\text {st }}$ October 2018 from this service.

Methods Descriptive analysis of routine data from registered users, including general demographics, service metrics of test kits (including nuclear acid amplification testing of Chlamydia and Gonorrhoea and/or blood serology), infection results and outcomes of kits (returned by $18^{\text {th }}$ January 2019).

Results 82,806 registered users ordered 81,542 kits. $80.3 \%$ $(n=65,460 / 81,542)$ kits were returned from 51,039 unique users. Proportion of sufficient samples in return kits: blood samples $=77.24 \% \quad(\mathrm{n}=50482 / 65361)$, vaginal samples $=$ $99.41 \% \quad(\mathrm{n}=38312 / 38541)$ and urine samples $=99.15 \%$ $(n=26595 / 26822)$. Median age 27 years old (range 16-99). Sex and sexual orientation demographics of unique users (n): Heterosexual: female $=54 \%$ (27560), male $=27.13 \%$ (13848), trans* $=0.05 \%$ (24) Homosexual: female $=0.45 \%$ (232), male $=11.56 \%$ (5898), trans* $=0.03 \%$ (14) Bisexual: female $=4.29 \%$ (2190), male $=2.41 \%$ (1231), trans ${ }^{*}=$ $0.08 \%$ (42) Reactive results from sufficient samples (numerator/denominator): Chlamydia 4.39\% (2850/64907), Gonorrhoea 1.12\% (726/64907), HIV 0.35\% (173/49889), Syphilis $0.58 \%$ (283/48692), Hepatitis B $0.73 \% \quad(n=69 / 9430)$ and Hepatitis C $0.56(n=54 / 9635) .100 \%$ of patients with a reactive HIV result have been contacted by a health advisor. 119 patients were considered 'low level' or could not be confirmed due to low sample volume. Of 54 'high level' reactive results, 32 were true positives and 18 patients were new positive patients. Median time (days) from requesting kit to kit testing was 9 days (range 1- 280 days). 99.3\% of reactive results were communicated within three days of receiving the sample. $97.2 \%(n=4454)$ of reactive Chlamydia results were confirmed to have transferred care to a clinic.

Conclusion These results provide an indication of service usage and outcomes of sexual health testing using online services.

Disclosure No significant relationships. 